noted, however, that the effect of FAAH inhibitors may not be entirely due to elevated levels of anandamide, as they also elevate the level of noncannabinoid substrates such as oleoylethanolamide ${ }^{6}$ or prostamides generated via COX-2 (ref. 7).

Historically, marijuana has been used to treat diarrhea and has been advocated for the treatment of a variety of other gastrointestinal problems, including Crohn's disease ${ }^{3}$. More recent pharmacological studies have clearly established that cannabinoids inhibit gastrointestinal motility and secretion by acting on $\mathrm{CB} 1$ receptors located on the terminals of both intrinsic and extrinsic submucosal neurons ${ }^{8}$. When administered to mice with chemically induced enteritis, cannabinoids also reduce inflammation ${ }^{9}$ and fluid accumulation ${ }^{10}$ in the gut. In these latter studies, high levels of anandamide and 2-arachidonoylglycerol as well as increased expression of $\mathrm{CB1}$ receptors have been detected in the inflamed intestines.

The novelty in the findings of Massa et al. ${ }^{3}$ is the active protective role of the endocannabinoid system, as indicated by the altered inflammatory response of mice lacking $\mathrm{CB} 1$ receptors or FAAH. Furthermore, CB1 activation reversed the electrophysiological signs of smooth muscle irritability and, at the same time, blunted the increase in tissue myeloperoxidase activity, a measure of leukocyte infiltration. These observations suggest that endocannabinoids protect the gut not only by decreasing bowel motility but also by inhibiting the inflammatory process itself.

The nature of the anti-inflammatory effect of endocannabinoids, however, remains to be elucidated. The exaggerated increase in myeloperoxidase activity in the inflamed bowel of CB1 knockout mice ${ }^{3}$ indicates that in wild-type mice endocannabinoids actively inhibit leukocyte infiltration caused by the chemical treatment. This may be due to inhibition of the release of chemokines and proinflammatory cytokines, such as TNF $\alpha$. TNF $\alpha$ has been implicated in the pathogenesis of $\mathrm{IBD}^{11}$, its likely source being activated macrophages and mast cells ${ }^{12}$. Cannabinoids suppress TNFo release from both cell types ${ }^{13,14}$ (although the CB1 antagonist SR141716 was also reported to suppress TNF $\alpha$ release in a different model of bowel injury ${ }^{15}$ ). In a mouse model of myocardial ischemia-reperfusion injury, cannabinoids attenuated the increase in myeloperoxidase activity via $\mathrm{CB} 2$ receptors ${ }^{16}$. The anti-inflammatory potential of CB2 receptors in the gut should therefore be explored.

Infiltrating macrophages in the inflamed bowel may be not only the target but also the source of endocannabinoids. Bacterial components, such as lipopolysaccharide (LPS), are known to powerfully induce anandamide synthesis in macrophages through a pathway dependent on CD14 and $\mathrm{NF}-\kappa \mathrm{B}^{5}$. Anandamide may thus act as an autocrine-paracrine mediator to limit the release of cytokines by macrophages and mast cells and the neuronal release of the tachykinins and acetylcholine that control bowel motility (Fig. 1).

An important feature of IBD is the altered tissue response to enteric bacteria. Mutations in the gene CARD15 (also called NOD2), which result in deficient activation of NF- $\mathrm{KB}$ in response to LPS, are associated with susceptibility to Crohn's disease ${ }^{17}$. A defect in $\mathrm{NF}-\kappa \mathrm{B}$ signaling is also expected to result in deficient activation of anandamide production given that NF- $\kappa B$ is required for LPSinduced anandamide synthesis ${ }^{5}$. Bowel inflammation can also increase FAAH activity $^{8}$, resulting in further reductions in anandamide levels.

It is not known whether anandamide levels are reduced and the activity of FAAH increased in the intestines of patients with IBD, but this could be verified using biopsy specimens. If confirmed, such changes may be interpreted as the weakening of an endogenous protective mechanism, which could be restored by preventing the breakdown of anandamide with a FAAH inhibitor. Biopsy results from humans could further support the case made by Massa et al. for targeting the endocannabinoid system to treat IBD, offering renewed hope to a much-suffering patient population.

1. Loftus, E.V. Gastroenterology 126, 1504-1517 (2004).

2. Longobardi, T., Jacobs, P. \& Bernstein, C.N. Am. J. Gastroenterol. 98, 1064-1072 (2003).

3. Massa, F. et al. J. Clin. Invest. 113, 1202-1209 (2004).

4. Cravatt, B.F. et al. Nature 384, 83-87 (1996).

5. Liu, J. et al. J. Biol. Chem. 278, 45034-45039 (2003).

6. Kathuria, S. et al. Nat. Med. 9, 76-81 (2003).

7. Weber, A. et al. J. Lipid Res. 45, 757-763 (2004).

8. Hornby, P.J. \& Prouty, S.M. Br. J. Pharmacol. 141, 1335-1345 (2004)

9. Izzo, A.A. et al. Br. J. Pharmacol. 134, 563-570 (2001).

10. Izzo, A.A. et al. Gastroenterology 125, 765-774 (2003).

11. Palladino, M.A., Bahjat, F.R., Theodorakis, E.A. \& Moldawer, L.L. Nat. Rev. Drug Discov. 2, 736-746 (2003).

12. Bischoff, S.C. et al. Gut 44, 643-652 (1999)

13. Samson, M.-T. et al. J. Immunol. 170, 4953-4962 (2003).

14. Fachinetti, F., Del Giudice, E., Furegato, S., Passarotto, M. \& Leon, A. Glia 41, 161-168 (2003).

15. Croci, T., Landi, M., Galzin, A.-M. \& Marini, P. Br. J. Pharmacol. 140, 115-122 (2003).

16. Di Filippo, C., Rossi, F., Rossi, S. \& D’Amico, M. J. Leukocyte Biol. 75, 453-459 (2004).

17. Hugot, J.-P. et al. Nature 411, 599-603 (2001).

\title{
APCs size up antigens
}

\section{Keith S Bahjat \& Stephen P Schoenberger}

\section{Intracellular antigens-from viruses, tumors, bacteria and other sources-pose a challenge to the immune response and to vaccine development. How do such antigens enter antigen-presenting cells? Three new studies examine this process, implicating whole proteins instead of small peptides as key intermediaries.}

Many of the pathogens that threaten human health do so from within the protected environment of our own cells and tissues. An effective immune response against such invaders often necessitates the drastic step of killing the host cells that harbor them. Cytotoxic $\mathrm{CD}^{+}{ }^{+} \mathrm{T}$ lymphocytes (CTLs) are especially suited to this task, with an impressive range of methods

Keith S Bahjat and Stephen P Schoenberger are at the La Jolla Institute for Allergy and Immunology, Division of Cellular Immunology, 10355 Science Center Drive, San Diego, California 92121, USA. e-mail: sps@liai.org for inducing the death of target cells. CTLs recognize target cells through major histocompatibility (MHC) class I molecules occupied with 8-10-amino-acid peptides derived from endogenous proteins. Initiation of CTL responses against infected target cells generally requires that the antigens presented at their surface must first be acquired and presented to CTLs by host antigen-presenting cells (APCs), such as dendritic cells. This poorly understood pathway of antigen trafficking and presentation is called 'cross-priming' and is essential for the generation of $\mathrm{CD}^{+} \mathrm{T}$-cell responses against viruses, intracellular bacteria, tumors and tissue antigens ${ }^{1,2}$. 
A central question in the immunobiology of cross-priming concerns the nature of the antigenic material transferred from donor cells to APCs; information about this would significantly influence vaccine design, immunotherapy and transplantation. Three recent papers reveal that cellular proteins, as opposed to the smaller peptides they contain, constitute the 'molecular currency' of cross-priming and have begun to shed light on the rules that govern this process $^{3-5}$.

APCs acquire foreign antigens by migrating to sites of inflammation; there they sample their microenvironment before moving on to lymphoid organs where their cargo of acquired antigens is displayed to responding $\mathrm{T}$ cells as smaller peptides bound to surface class I MHC molecules. One attractive theory holds that peptides are transferred to APCs as extracellular complexes bound to cellular heat shock proteins (HSPs) ${ }^{6}$. In support of this idea, HSP complexes containing specific antigenic peptides can be isolated from variety of cell types and taken up by specific receptors into APCs in vitro; on in vivo administration, these APCs can crossprime $\mathrm{CD}^{+}{ }^{+}$T cells ${ }^{7}$.

Another hypothesis states that cellular antigens are transferred to APCs as intact proteins or large fragments taken up through phagocytosis ${ }^{8}$. Understanding the relative contribution of these pathways to cross-priming is more than an academic matter; cell-associated antigen activates $\mathrm{CD}^{+} \mathrm{T}$ cells in vivo much more efficiently than does soluble antigen, raising the possibility that vaccination strategies based on exploiting the cross-priming pathway could promote superior T-cell responses ${ }^{9}$.

The new studies, by Norbury et al. ${ }^{4}$, Shen et al. ${ }^{5}$ and Wolkers et al. ${ }^{3}$, take a common approach to this question, examining the ability of donor cells expressing different forms of a precursor antigen to generate $\mathrm{CD}^{+}$T-cell responses through crosspriming. Norbury et al. used recombinant vaccinia virus to transduce MHC-mismatched tumor cells with various forms of ovalbumin. These forms included a minimal OVA $257-264$ epitope targeted to either the cytoplasm or the endoplasmic reticulum and expressed within either a larger chimeric fusion protein or the full-length OVA protein itself. Each construct could prime $\mathrm{OVA}_{257-264^{-}}$specific $\mathrm{CD}^{+} \mathrm{T}$ cells after direct infection of mice. When the cells expressing the various constructs were used for immunization, however, only the larger forms of the source antigen (intact

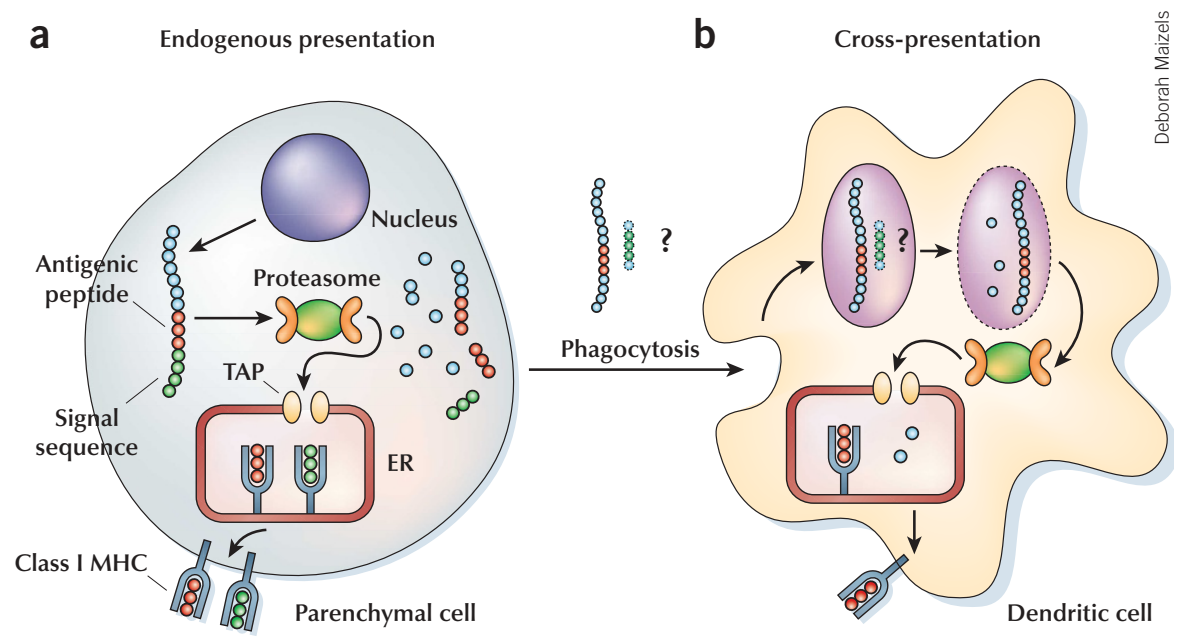

Figure 1 The long and short of cross-priming. Parenchymal cells (left) can generate surface complexes of class I MHC molecules occupied with virtually any cellular peptide of the correct length and sequence, including those found within a mature protein (red fragment) or derived from a signal sequence (green fragment). Cross-priming involves the uptake of cellular antigens by bone marrow-derived APCs (right), which are then directed to an endogenous class I MHC presentation pathway. The three new studies indicate that it is mature proteins, instead of smaller peptides, that are transferred from donor cell to APCs during cross-priming and suggest a bias against epitopes contained within signal sequences in this process. ER, endoplasmic reticulum.

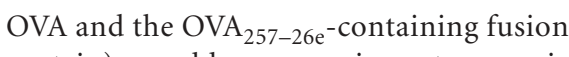
protein) could cross-prime transgenic $\mathrm{OVA}_{257-264}$-specific CD8 ${ }^{+} \mathrm{T}$ cells.

Norbury et al. then examined crosspriming of $\mathrm{T}$ cells by a rapidly degradable form of the epitope-containing fusion protein. The fusion protein could cross-prime $\mathrm{T}$ cells only if donor cells were first treated with a proteasome inhibitor, although the same treatment did not boost the ability of cells expressing stable $\mathrm{OVA}_{257-264}$-containing proteins to cross-prime OVA-specific CTLs.

The results of Norbury et al. indicate that cross-priming is not mediated by exogenous or endogenous peptides but rather through the transfer of intact proteins (possibly through phagocytosis) or protein fragments from donor cells to APCs. These findings dovetail with those of Shen et al., who found that the ability of an antigen to cross-prime $\mathrm{CD} 8^{+} \mathrm{T}$ cells did not depend on colocalization with heat shock proteins but rather on the amount of intact source protein and its subcellular location (cytoplasm, endoplasmic reticulum or plasma membrane).

Wolkers et al. asked whether the location of an antigenic peptide within its source protein could influence cross-priming of $\mathrm{CD}^{+} \mathrm{T}$ cells. Using green fluorescent protein (GFP) as a model antigen, the authors engineered several chimeric proteins. They placed one of two distinct CD8 ${ }^{+}$T-cell epitopes either near the $\mathrm{N}$-terminus, within a signal sequence, or near the C-terminus of GFP. Using both $\mathrm{T}$-cell recognition and biochemical assays, the authors showed that when these constructs were expressed in wild-type tumor cells, both epitopes were presented with comparable efficiency irrespective of their position within the chimeric GFP molecules.

The authors then expressed these constructs in tumor cells lacking the transporter associated with antigen processing (TAP), a heterodimeric complex that translocates cytosolic peptides into the endoplasmic reticulum. Immunization of mice with these TAP-deficient cells, a setting that depends on cross-priming, could elicit antigen-specific $\mathrm{CD}^{+} \mathrm{T}$ cells only when the relevant epitope was located in the C-terminal position of the chimeric protein. These data support the idea that cross-priming favors antigenic peptides located within mature proteins and reveal an unexpected bias against epitopes located within functional signal peptides.

Each of these studies square nicely with two recent reports describing a pathway in antigen-presenting dendritic cells for endocytosed material (presumably including intact proteins); this pathway leads from the phagosome to the cytosol and results in protein degradation and presen- 
tation via the endogenous class I MHC machinery (Fig. 1) ${ }^{10,11}$. The finding that the location of an epitope within a larger protein can profoundly influence crosspriming of $\mathrm{CD}^{+} \mathrm{T}$ cells may help to clarify some of the controversy surrounding the ability of different antigens to participate in this process ${ }^{12}$

Taken together, these three new studies provide a useful theoretical foundation for understanding the cross-priming pathway, and offer a practical framework for its exploitation. If cellular proteins and not peptides are the source material for crosspriming of $\mathrm{CD}^{+} \mathrm{T}$ cells, vaccination strategies based on maximizing steadystate expression levels and stability in donor cells would be predicted to generate superior $\mathrm{CD}^{+} \mathrm{T}$ cell responses. Further studies will certainly shed light on this and perhaps allow the meaningful translation of this new understanding from bench to bedside.

1. den Haan, J.M. \& Bevan, M.J. Curr. Opin. Immunol. 13, 437-441 (2001).

2. Heath, W.R. \& Carbone, F.R. Annu. Rev. Immunol.
19, 47-64 (2001).

3. Wolkers, M.C. et al. Science published online 28 May 2004 (doi: 10.1126/science.1096268).

4. Norbury, C.C. et al. Science published online 28 May 2004 (doi: 10.1126/science.1096378).

5. Shen, L. \& Rock, K.L. Proc. Natl. Acad. Sci. USA 101, 3035-3040 (2004).

6. Suto, R. \& Srivastava, P.K. Science 269, 1585-1588 (1995).

7. Srivastava, P.K. \& Amato, R.J. Vaccine 19, 2590-2597 (2001).

8. Rock, K.L. Immunol. Today 17, 131-137 (1996).

9. Li, M. et al. J. Immunol. 166, 6099-6103 (2001).

10. Guermonprez, P. et al. Nature 425, 397-402 (2003).

11. Houde, M., et al. Nature 425, 402-406 (2003).

12. Zinkernagel, R.M. Eur. J. Immunol. 32 , 2385-2392 (2002).

\title{
Cellular energy sensor balances the scales
}

\author{
Caroline J Small, David Carling \& Stephen R Bloom
}

\section{Treatment with $\alpha$-lipoic acid, found naturally in our diets, decreases food intake in rats and keeps them slim by inhibiting AMP activated protein kinase (AMPK) in the hypothalamus. The findings reinforce the emerging status of AMPK as a cellular integrator that senses signals for food intake and whole body energy status (pages 727-733).}

\begin{abstract}
Those of us who must regularly delete 'spam' from their e-mail systems may be familiar with $\alpha$-lipoic acid. Its use as a dietary supplement is touted on the Internet as a cure for ailments ranging from cataracts to chronic fatigue syndrome and Alzheimer disease (and all for the bargain price of $\$ 29.70$ for 120 tablets; Fig. 1). Although these claims are dubious, $\alpha$ lipoic acid is known to have antioxidant properties and acts as a cofactor for mitochondrial dehydrogenases.

In this issue, Kim et al. ${ }^{1}$ give the makers of supplements something else to chew on: they show that $\alpha$-lipoic acid, given either as part of the diet or by intraperitoneal or intracerebroventricular (i.c.v.) injection, leads to decreased food intake and body weight in rats.
\end{abstract}

The hypothalamus coordinates signals from peripheral tissues, such as the gut and fat, to control appetite. The adiposederived hormone leptin, as well as gut hor-

Caroline J. Small and Stephen R. Bloom are at the Endocrine Unit, and David Carling is at the Endocrine Unit and the MRC Clinical Sciences Centre Cellular Stress Group, Imperial College London, Hammersmith Campus, Du Cane Road, London W12 ONN, UK.

e-mail: dcarling@imperial.ac.uk or

s.bloom@imperial.ac.uk mones including peptide YY (PYY), glucagon-like peptide-1 and oxyntomodulin, signal to the hypothalamus to decrease feeding. In contrast, Ghrelin, which is produced in the stomach, signals to the hypothalamus to increase feeding. The identification of common pathways for these regulators in the hypothalamus is crucial for understanding potential therapeutic interventions to treat obesity, a dire health problem in many countries. The World Health Organization has recently predicted that diabetes, atherosclerosis and malignancy will double over the next 20 years as a consequence of increasing obesity.

Kim et al. examined the effect of $\alpha$-lipoic acid on whole-body energy metabolism and found that it alters the activity of a molecule that is emerging as a central regulator of energy balance, AMPK. In addition to decreased food intake, the authors show that rats treated with $\alpha$-lipoic acid lost more body weight than pair-fed untreated controls, suggesting an increase in energy expenditure. They go onto show that $\alpha$ lipoic acid caused an increase in the expression of uncoupling protein-1 (Ucp1) mRNA in brown adipose tissue. Ucp 1 is a member of a family of mitochondrial membrane proteins that are thought to allow protons to leak into the mitochondrial matrix, dissipating the proton-motive force as heat.
An obvious interpretation of the data is that the increased level of Ucp 1 in response to $\alpha$-lipoic acid increases overall energy expenditure by oxidizing fat stores. Kim et al. also report that pharmacological activation of AMPK in the hypothalamus by i.c.v. injection of 5-aminoimidazole-4-carboxamide ribose (AICAR) abolished the increase in energy expenditure in response to $\alpha$-lipoic acid as well as the increase in Ucp 1 expression in brown adipose tissue. However, without knowing the effect of AICAR administration alone on energy expenditure, it is difficult to fully interpret these results.

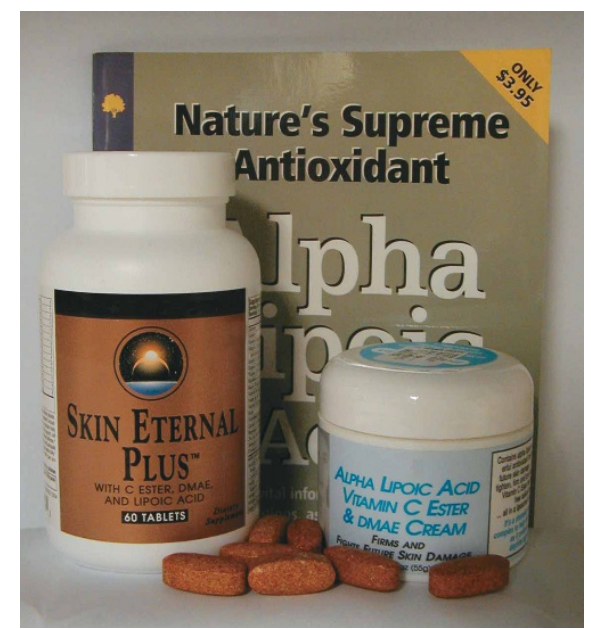

Figure 1 AMPK inhibitor 